\title{
Impaired Expected Value Computations in Schizophrenia Are Associated With a Reduced Ability to Integrate Reward Probability and Magnitude of Recent Outcomes
}

Citation for published version (APA):

Hernaus, D., Frank, M. J., Brown, E. C., Brown, J. K., Gold, J. M., \& Waltz, J. A. (2019). Impaired Expected Value Computations in Schizophrenia Are Associated With a Reduced Ability to Integrate Reward Probability and Magnitude of Recent Outcomes. Biological Psychiatry: Cognitive Neuroscience and Neuroimaging, 4(3), 280-290. https://doi.org/10.1016/j.bpsc.2018.11.011

Document status and date:

Published: 01/03/2019

DOI:

10.1016/j.bpsc.2018.11.011

Document Version:

Publisher's PDF, also known as Version of record

\section{Document license:}

Taverne

\section{Please check the document version of this publication:}

- A submitted manuscript is the version of the article upon submission and before peer-review. There can be important differences between the submitted version and the official published version of record. People interested in the research are advised to contact the author for the final version of the publication, or visit the DOI to the publisher's website.

- The final author version and the galley proof are versions of the publication after peer review.

- The final published version features the final layout of the paper including the volume, issue and page numbers.

Link to publication

\footnotetext{
General rights rights.

- You may freely distribute the URL identifying the publication in the public portal. please follow below link for the End User Agreement:

www.umlib.nl/taverne-license

Take down policy

If you believe that this document breaches copyright please contact us at:

repository@maastrichtuniversity.nl

providing details and we will investigate your claim.
}

Copyright and moral rights for the publications made accessible in the public portal are retained by the authors and/or other copyright owners and it is a condition of accessing publications that users recognise and abide by the legal requirements associated with these

- Users may download and print one copy of any publication from the public portal for the purpose of private study or research.

- You may not further distribute the material or use it for any profit-making activity or commercial gain

If the publication is distributed under the terms of Article 25fa of the Dutch Copyright Act, indicated by the "Taverne" license above, 


\title{
Impaired Expected Value Computations in Schizophrenia Are Associated With a Reduced Ability to Integrate Reward Probability and Magnitude of Recent Outcomes
}

\author{
Dennis Hernaus, Michael J. Frank, Elliot C. Brown, Jaime K. Brown, James M. Gold, and \\ James A. Waltz
}

\begin{abstract}
BACKGROUND: Motivational deficits in people with schizophrenia (PSZ) are associated with an inability to integrate the magnitude and probability of previous outcomes. The mechanisms that underlie probability-magnitude integration deficits, however, are poorly understood. We hypothesized that increased reliance on "valueless" stimulus-response associations, in lieu of expected value (EV)-based learning, could drive probability-magnitude integration deficits in PSZ with motivational deficits.

METHODS: Healthy volunteers $(n=38)$ and PSZ $(n=49)$ completed a learning paradigm consisting of four stimulus pairs. Reward magnitude $(3,2,1,0$ points) and probability $(90 \%, 80 \%, 20 \%, 10 \%)$ determined each stimulus's EV. Following a learning phase, new and familiar stimulus pairings were presented. Participants were asked to select stimuli with the highest reward value.

RESULTS: PSZ with high motivational deficits made increasingly less optimal choices as the difference in reward value (probability $\times$ magnitude) between two competing stimuli increased. Using a previously validated computational hybrid model, PSZ relied less on EV ("Q-learning") and more on stimulus-response learning ("actor-critic"), which correlated with Scale for the Assessment of Negative Symptoms motivational deficit severity. PSZ specifically failed to represent reward magnitude, consistent with model demonstrations showing that response tendencies in the actor-critic were preferentially driven by reward probability.

CONCLUSIONS: Probability-magnitude deficits in PSZ with motivational deficits arise from underutilization of EV in favor of reliance on valueless stimulus-response associations. Confirmed by our computational hybrid framework, probability-magnitude integration deficits were driven specifically by a failure to represent reward magnitude. This work provides a first mechanistic explanation of complex EV-based learning deficits in PSZ with motivational deficits that arise from an inability to combine information from different reward modalities.
\end{abstract}

Keywords: Anhedonia, Basal ganglia, Dopamine, Orbitofrontal cortex, Reinforcement learning, Schizophrenia https://doi.org/10.1016/j.bpsc.2018.11.011

Many people with schizophrenia (PSZ) suffer from a reduced tendency to engage in goal-directed behavior $(1,2)$, termed amotivation or avolition. These deficits in motivation can contribute substantially to poor functional capacity and quality of life (3-5). One explanation suggests that motivational deficits result from a specific impairment in the ability to precisely represent the value of an action or choice (expected value [EV]) coupled with overreliance on "valueless" stimulus-response associations (6-8). Support for this computational account, however, comes from studies using reinforcement learning $(\mathrm{RL})$ paradigms in which reward probability (the chance of obtaining a reward) solely determines EV. Importantly, other evidence indicates that EV estimation deficits in PSZ (with motivational deficits) are most prominent when EV depends on the successful integration of reward magnitude (size) and probability.

One important line of evidence suggesting prominent deficits in EV representation comes from the lowa Gambling Task (9), in which participants select from four card decks with varying reward magnitude and probability. Performance deficits on this task, in PSZ, are driven by a reduced ability to integrate long-term outcome magnitude and probability (10-12). These deficits extend to contexts in which participants choose between earning a small certain reward or gamble for a larger reward (a "framing" task) (13). When reward magnitude and probability vary continuously, impaired EV computations in PSZ with motivational deficits are primarily driven by decreased sensitivity to reward magnitude (14). In contrast, 
PSZ can optimize task performance eventually if they learn about reward probability (15). Thus, the available evidence suggests that probability-magnitude integration deficits in PSZ (with motivational deficits) may be driven by impaired sensitivity to reward magnitude specifically, and by somewhat spared tracking of reward probability. To date, however, the computational mechanisms associated with an inability to integrate reward probability and magnitude into the estimation of EV has never been investigated. The goal of the current study was to use computational modeling to provide a mechanistic account of probability-magnitude integration deficits in PSZ.

There are several approaches to $\mathrm{RL}$ in computational models, which may in turn relate to different cognitive and neural mechanisms. While all $\mathrm{RL}$ models involve the reward prediction error (RPE) as a critical quantity that drives learning, the way in which the RPE is calculated and used to optimize behavior differs among classes. In the Q-learning framework (16), RPEs are used to update the EV of every action (here, choosing a stimulus), and choices are then executed directly based on the relative EV estimate. Computational models and neural data suggest that the dynamic representation of EV-specifically, the integration of reward magnitude and probability-crucially involves the orbitofrontal cortex (OFC) (17-22). In the actor-critic framework (23), however, RPEs are not used to directly update choice EV. Instead, the direct actor develops action propensities in a valueless space; choice preferences are only indirectly related to RPEs generated by the critic. One potential mechanism involved in the formation of such stimulus-response associations is thought to be the gradual tuning of basal ganglia (BG) synaptic weights in response to dopaminergic RPEs $(24,25)$.

Although the differences between these two model classes may seem subtle, and both algorithms will generally produce adaptive learning, they can make categorically different predictions in particular scenarios. For example, since the actor only relies on choice propensities, given a novel choice between an option that had yielded gains and another one that had merely avoided losses, the actor will not exhibit a preference for the higher EV option if both options had given rise to positive RPEs in the critic. Using a hybrid computational model that allows for parametric mixing between Q-learning and actor-critic learning, we have previously shown that decreased reliance on the former and a relative increase in the latter can account for decreased gain-seeking behavior and overvaluation of contextual information in PSZ with motivational deficits $(6,7)$, both of which result in a poor representation of EV. While we have argued previously, using this approach, that decreased reliance on EV and overutilization of stimulus-response associations serves as one computational framework of impaired goaldirected behavior, the ability of this framework to provide a mechanistic explanation of complex probability-magnitude integration deficits has never been assessed $(6,7)$. Moreover, probability-magnitude integration deficits in PSZ provide a unique opportunity to assess the generalizability of the computational hybrid framework to other EV estimation deficits.

In the current study, we hypothesized that the hybrid computational framework, via a reduction in Q-learning relative to actor-critic-type learning, can potentially account for abnormal probability-magnitude integration. First, because actor weights increase with each critic RPE, in stochastic environments they do not converge to a true expected value, and as such they are overly influenced by frequency. Second, neural network models of OFC and BG (26) have suggested that dysfunction of the OFC can bias BG choices to be primarily driven by reward probability, whereas OFC integrity was needed to improve EV estimation by reward magnitudes. Similar probability patterns have been observed in algorithmic versions of this BG model, as in the Opponent Actor Learning architecture, which is a modified actor-critic based on physiological properties of the BG (25). To the degree that Q-learning and actor-critic capture OFC- and BG-like properties, we thus hypothesized that such a probability bias might be captured by our hybrid computational model in terms of an overreliance on actor-critic versus Q-learning.

In the study reported here, participants were presented with an $\mathrm{RL}$ task in which they learned to select stimuli with the highest reward value. Pairs of stimuli differed in both reward probability and magnitude. In a subsequent transfer phase, old and novel pairs of stimuli were presented. Optimal performance in this phase of the task crucially depended on one's ability to integrate reward probability and magnitude, which is different from more common RL paradigms, where $\mathrm{EV}$ is a function of reward probability only.

Through computational modeling and statistical analyses, we tested for the first time whether probability-magnitude integration deficits in PSZ (with motivational deficits) could be explained by underutilization of EV and/or overreliance on stimulusresponse associations. In line with previous work $(6,7,27)$, we predicted that deficits in the representation of EV should become more apparent as choices become easier. That is, we predicted that performance differences between PSZ and healthy volunteers would be largest when the difference in EV between two competing stimuli was greatest (note that greater deficits with increasing difficulty would point to a more general learning impairment). Moreover, we expected that probabilitymagnitude integration deficits in PSZ, as well as reliance on reward probability versus magnitude in the entire sample, would correlate with the degree to which individuals relied on Q-learning versus actor-critic type learning. Finally, we expected probability-magnitude deficits and computational evidence thereof to correlate with the severity of motivational deficits.

\section{METHODS AND MATERIALS}

\section{Participants}

Our study included 49 participants with a diagnosis of schizophrenia or schizoaffective disorder (PSZ) and 38 healthy volunteers (HVs) who did not differ on, among other factors, age, gender, and ethnicity (Table 1). Inclusion criteria and cognitive and clinical assessment details are reported in the Supplement (see "Cognitive and Clinical Assessments," including cutoff scores for less severe motivational deficit [LMD] and more severe motivational deficit [MMD] subgroups). Written informed consent was obtained from all participants prior to the experiment.

\section{Probability-Magnitude Integration Task}

Participants completed a probabilistic stimulus selection task consisting of a learning phase (160 trials) and test/transfer 
Table 1. Sample Demographics

\begin{tabular}{|c|c|c|c|c|}
\hline & $\begin{array}{c}\mathrm{HVs} \\
(n=38)\end{array}$ & $\begin{array}{c}\text { PSZ } \\
(n=49)\end{array}$ & $t / \chi^{2}$ & $p$ \\
\hline Age, Years & $37.16(12.65)$ & $38.63(10.58)$ & -0.59 & .56 \\
\hline Gender (F, M), $n$ & 13,25 & 16,33 & 0.02 & .88 \\
\hline $\begin{array}{l}\text { Race (African American, } \\
\text { Caucasian, other), } n\end{array}$ & $15,21,2$ & $19,26,4$ & 0.28 & .87 \\
\hline Education Level, Years $^{a}$ & $14.94(1.97)$ & $13.06(2.07)$ & 4.23 & $<.001$ \\
\hline Maternal Education Level & $14.57(2.16)$ & $13.48(2.44)$ & 2.10 & .04 \\
\hline Paternal Education Level & $14.40(2.90)$ & $13.37(3.60)$ & 1.38 & .17 \\
\hline WTAR IQ & $112.18(10.59)$ & $97.47(16.97)$ & 4.68 & $<.001$ \\
\hline WASI IQ & $117.47(8.67)$ & $100.69(13.84)$ & 6.54 & $<.001$ \\
\hline \multicolumn{5}{|l|}{ MATRICS Domains } \\
\hline Processing speed & $55.26(8.71)$ & $37.55(13.21)$ & 7.14 & $<.001$ \\
\hline Attention/vigilance & $53.21(7.58)$ & $41.29(11.22)$ & 5.63 & $<.001$ \\
\hline Working memory & $50.92(9.25)$ & $39.47(10.50)$ & 5.31 & $<.001$ \\
\hline Verbal learning & $54.00(9.06)$ & $39.53(9.04)$ & 7.40 & $<.001$ \\
\hline Visual learning & $50.47(9.88)$ & $36.41(13.68)$ & 5.35 & $<.001$ \\
\hline Reasoning & $52.37(10.31)$ & $42.22(10.37)$ & 4.54 & $<.001$ \\
\hline Social cognition & $53.61(9.09)$ & $39.37(10.74)$ & 6.55 & $<.001$ \\
\hline MATRICS overall score & $54.32(8.00)$ & $32.71(12.55)$ & 9.25 & $<.001$ \\
\hline \multicolumn{5}{|l|}{ Antipsychotic Medication } \\
\hline Total haloperidol & - & $11.57(8.26)$ & - & - \\
\hline \multicolumn{5}{|l|}{ Clinical Ratings } \\
\hline BPRS positive, sum & - & $7.92(3.94)$ & - & - \\
\hline SANS AA/RF, sum & - & $16.16(8.14)$ & - & - \\
\hline SANS AFB/Alog, sum & & $11.02(7.83)$ & - & - \\
\hline
\end{tabular}

Values are mean (SD), except where noted.

$\mathrm{AA} / \mathrm{RF}$, avolition-apathy (including role-functioning) and asocialityanhedonia; AFB, affective flattening; Alog, alogia; BPRS, Brief Psychiatric Rating Scale; F, female; HVs, healthy volunteers; M, male; PSZ, people with schizophrenia; SANS, Scale for the Assessment of Negative Symptoms; WASI, Wechsler Abbreviated Scale of Intelligence; WTAR, Wechsler Test of Adult Reading.

${ }^{a}$ Education level missing for $2 \mathrm{HVs}$; maternal education level missing for 3 HVs and 2 PSZ; paternal education level missing for $3 \mathrm{HVs}$ and 3 PSZ.

phase (64 trials) (Figure 1). During each learning phase trial, two stimuli were presented, on either side of a fixation cross. Participants were prompted to select one stimulus by pressing either the left or right trigger on a gamepad using their left or right index finger. Each choice was followed immediately by feedback, in the form of a number of points $(+3,+2,+1$, or +0$)$. The eight stimuli differed in the probability and magnitude of the expected reward. For one pair, one stimulus resulted in a three-point win on $90 \%$ of all trials, and a zeropoint win on $10 \%$ of trials. The other option resulted in a three-point win on $10 \%$ of all trials, and a zero-point win on $90 \%$ of trials (90-10/3 pair). The other three pairs were a "90$10 / 1$ " (one-point win $90 \%$ of the time for the optimal stimulus, one-point win $10 \%$ of the time for the nonoptimal stimulus), "80-20/2" (two-point win $80 \%$ of the time for the optimal stimulus, two-point win $20 \%$ of the time for the nonoptimal stimulus), and " $80-20 / 1$ " (one-point win $80 \%$ of the time for the optimal stimulus, one-point win $20 \%$ of the time for the nonoptimal stimulus) pair. All pairs were presented 40 times in pseudorandomized order. Stimulus location (optimal stimulus on left or right side of screen) was randomized and counterbalanced across trials, and stimulus-value pairings were fully randomized across participants. Participants were informed that the aim of the task was to accumulate as many points as possible, and that they would be rewarded for good performance. All participants received the same monetary performance bonus at the end of the experiment.

The purpose of the test/transfer phase was to assess participants' ability to combine reward probability and magnitude into a representation of EV. Participants were presented with the four familiar learning phase pairs ("acquisition pairs"; four presentations per pair) and 24 novel pairs of stimuli (two presentations per pair) and received the following instructions: "Please choose the picture that feels like it's worth more points based on what you have learned during the previous block." Stimulus location was randomized across trials. Crucially, for many of these trials, the optimal answer depended on the ability to combine the expected probability and magnitude of a stimulus (e.g., $80 / 2$ vs. $90 / 1$, or $10 / 3$ vs. $20 / 2$ ). No performance feedback was presented during the test/transfer phase.

\section{Hybrid Computational Model}

Using 1) posterior predictive simulations (using the fitted parameters to simulate performance), 2) demonstrations of learning on the basis of reward magnitude and probability, and 3) quantifications of model evidence, the "hybrid-probability" model was most likely to be the optimal model. Crucially, in the hybrid-probability model, Q-learning had access to the reward magnitude of outcomes, while the actor-critic received only binary outcomes (i.e., zero or one point). Thus, in the hybridprobability model, learning in the actor-critic was restricted to reward frequency. Owing to space restrictions, we report all our computational model details (including rationale, simulations, model demonstrations, fitting procedure, and model evidence) in the Supplement.

\section{Statistical Analyses}

Effects of diagnostic group (PSZ vs. HV), trial block (four blocks of 10 trials), and choice pair (90-10/3, 80-20/2, 90-10/1, $80-20 / 1$ ) on acquisition pair performance were investigated using a repeated measures analysis of variance. Betweengroup differences in model parameters were ascertained using univariate analysis of variance and independent sample $t$ tests.

In accordance with previous work (7), novel stimulus pairs in the test/transfer phase were ranked using the difference in EV between two competing stimuli $(\mathrm{EV}=$ probability $\times$ magnitude $)$ (see Supplemental Table S1 for details regarding stimulus combinations). A value difference tracking slope was computed for every participant using a logistic regression with value difference (value left - value right) as predictor and button press (left $=1$, right $=0$ ) as a nuisance regressor (thereby correcting for the tendency to select one side of the screen over the other).

Exploratory analyses into trials that were matched for reward magnitude while differing in reward probability ("probability discrimination trials"; 90/1-80/1), and trials that were matched for reward probability while differing in reward magnitude ("magnitude discrimination trials"; 90/3-90/1 and 
160-trial Learning Phase

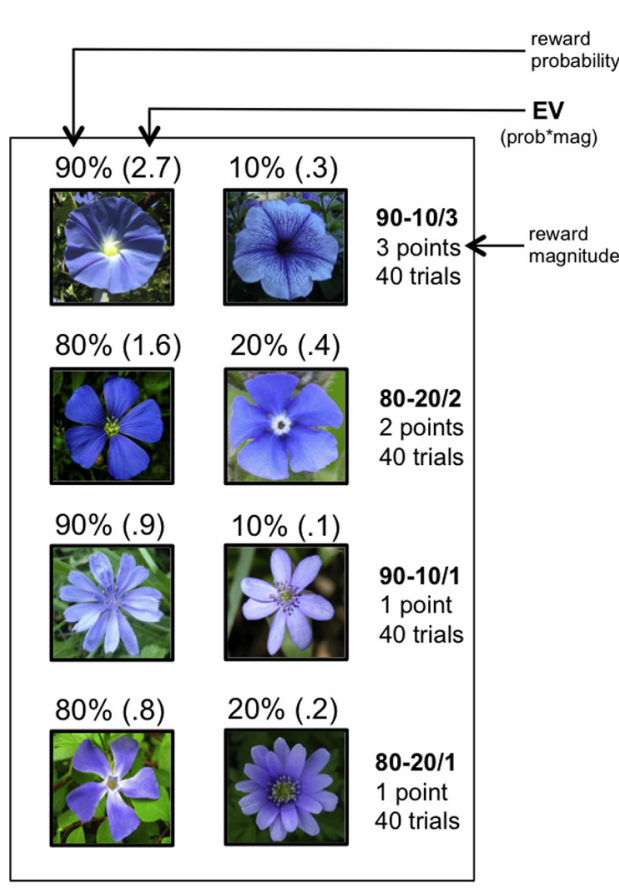

Trial Example

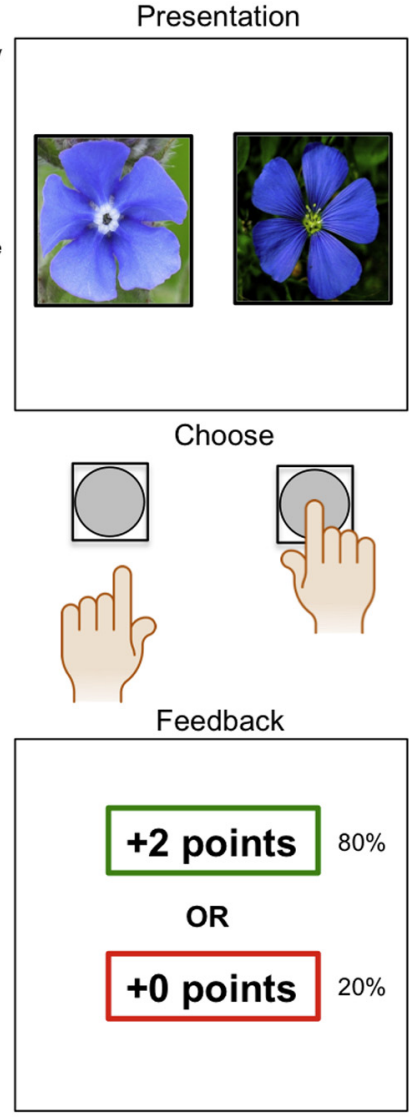

\section{4-trial Transfer Phase}
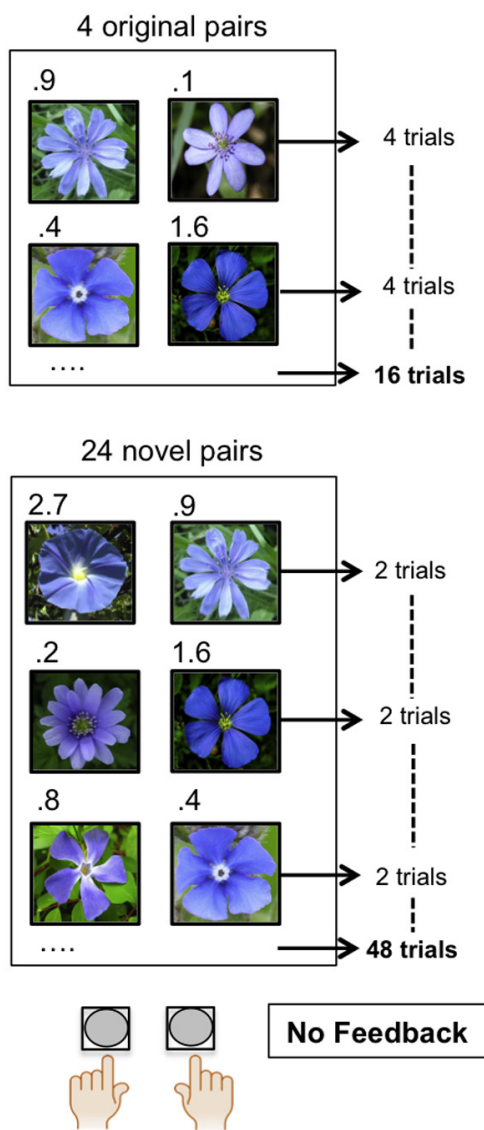

Figure 1. Graphical overview of the probability-magnitude integration task. EV, expected value; mag, magnitude; prob, probability.

80/2-80/1), were conducted, as well as a group $\times$ trial type (probability-magnitude discrimination) interaction. The latter analysis investigated whether performance advantages conferred by learning from reward magnitude versus learning from reward probability differed between the two diagnostic groups.

Correlation analyses were conducted using Spearman's coefficients (due to non-normal distributions of many variables). Key findings, including group differences in learning phase accuracy, value difference tracking slope, mixing parameter (HV vs. MMD), and correlations between the mixing parameter and test/transfer phase performance in the entire sample, all survived Bonferroni correction for the number of model parameters $(p=.01)$. Skewness and kurtosis of all parameters was within the $-2 /+2$ bounds, and HV versus MMD parameters differences remained when repeating analyses using a Mann-Whitney $U$ test.

\section{RESULTS}

\section{Demographics}

Participant groups did not differ on key demographic variables, including, age, gender, race, and paternal education, although
PSZ scored lower on measures of $I Q$ and all MATRICS Consensus Cognitive Battery subdomains (Table 1). LMD and MMD subgroups did not differ on IQ (Wechsler Test of Adult Reading: $t_{47}=0.35, p=.73$ ), MATRICS performance (overall: $\left.t_{47}=1.42, p=.17\right)$, antipsychotic dosage $\left(t_{47}=-1.04, p=.31\right)$, the Brief Psychiatric Rating Scale (BPRS) positive symptom factor $\left(t_{47}=-1.49, p=.14\right)$, or BPRS depression scores $\left(t_{47}=-0.59, p=.56\right)$.

\section{Performance on Acquisition Pairs}

In the learning phase, HVs compared with PSZ overall more often selected the optimal stimulus $\left(F_{1,85}=13.41, p<.001\right)$. There was no evidence for a group $\times$ trial block $\times$ pair $\left(F_{7.22,613.92}=0.44, p=.88\right)$, group $\times$ pair $\left(F_{2.68,228.01}=0.29, p=\right.$ $.81)$, or group $\times$ trial block $\left(F_{2.33,197.73}=0.48, p=.77\right)$ interaction. HVs outperformed PSZ on all stimulus pairs $(90-10 / 3$, $t_{85}=3.17, p=.002 ; 80-20 / 2 ; t_{85}=2.90, p=.005 ; 90-10 / 1 ; t_{85}=$ $1.90, p=.06 ; 80-20 / 1 ; t_{85}=2.62, p=.01$ ) (Figure 2A). Performance in block 4 (trials 31-40) was above chance in both participant groups for every pair (all $p<.001$ ). There was also a main effect of pair $\left(F_{2.69,231}=15.17, p<.001\right)$, with post hoc comparisons suggesting that both greater reward magnitude $\left(90-10 / 3\right.$ vs. $90-10 / 1, t_{86}=4.50, p<.001 ; 80-20 / 2$ vs. $80-20 / 1$, 
A

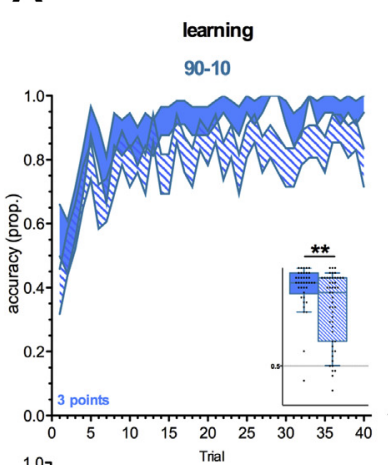

\section{Acquisition Phase Pairs}
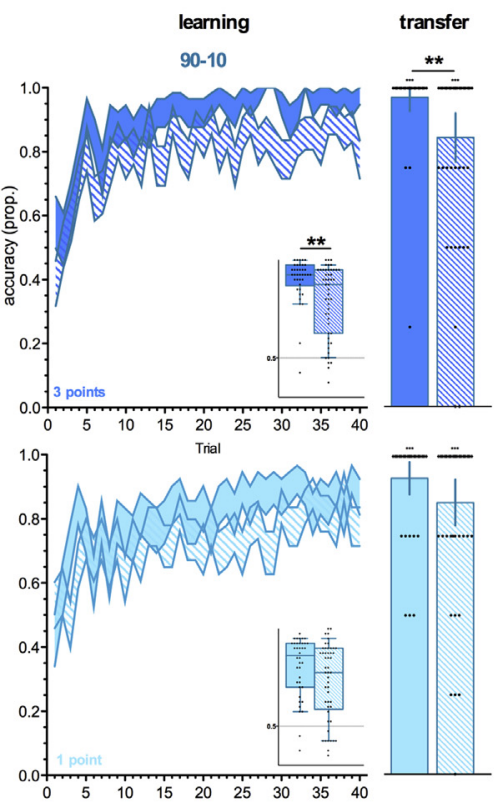

B

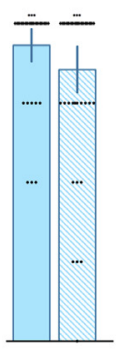

\section{Test/Transfer Phase}

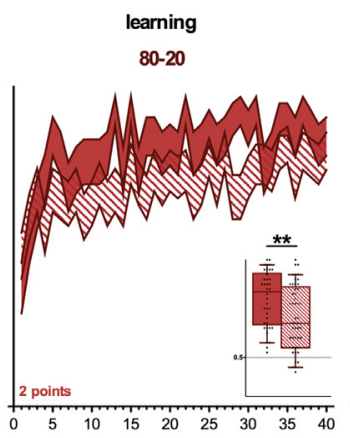

transfer
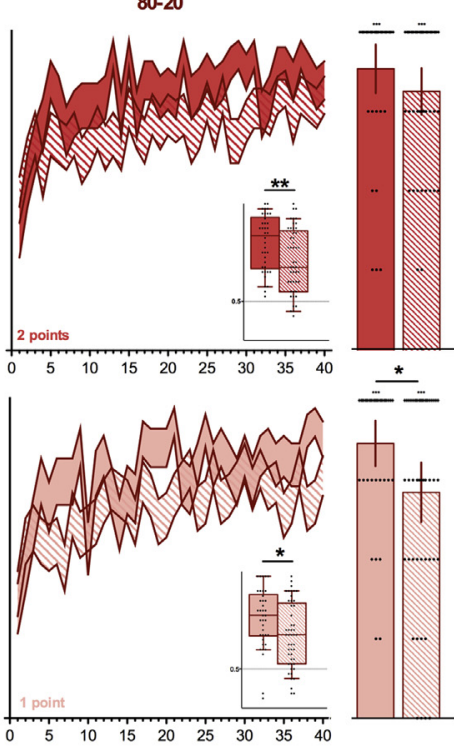

novel pairs
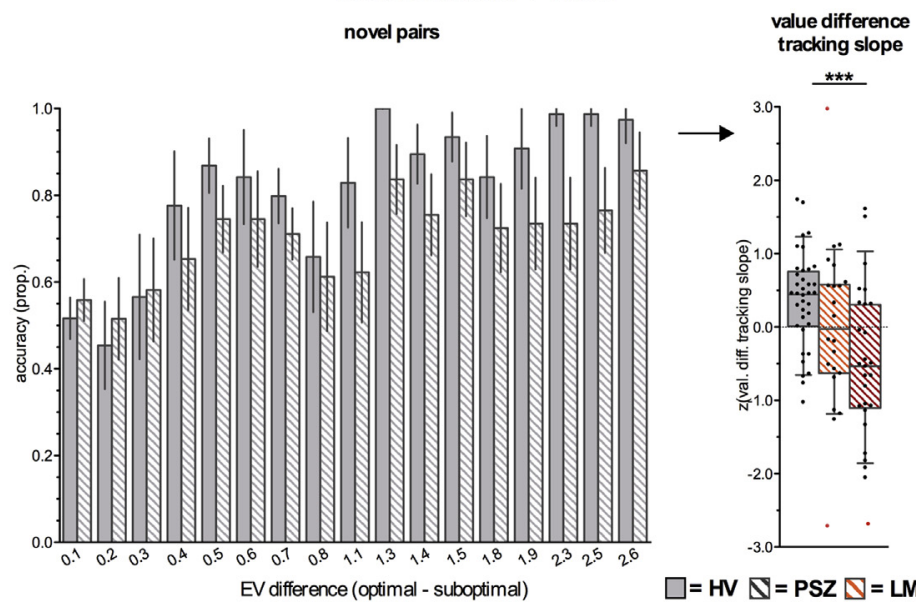

slope

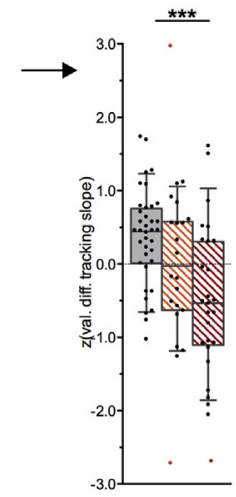

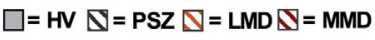

Figure 2. Performance on acquisition phase pairs. (A) Trial-by-trial (large figure) and average (small figure) learning phase accuracy (below "learning"), plotted next to average accuracy on acquisition phase pairs in the test/transfer phase (below "transfer"). (B) Test/transfer phase performance on novel pairs ranked on expected value (EV) (probability-by-magnitude) difference between two competing stimuli, once presented for every level of value difference separately ("novel pairs"), and once presented as the value difference tracking slope (for less severe motivational deficit [LMD] and more severe motivational deficit [MMD] separately; "value difference tracking slope"). Two LMD and one MMD were removed from these analyses due to limited choice variability, which produced extreme performance slopes (marked in red). ${ }^{*} p<.05,{ }^{\star \star} p<.01$ ${ }^{* *} p<.001$. Small asterisks above bars represent significance against chance. Error bars in panel (A) represent SEM and in (B) reflect 95\% confidence intervals. HVs, healthy volunteers; PSZ, people with schizophrenia. $\left.t_{86}=1.58, p=.12\right)$ and probability (90-10/1 vs. $80-20 / 1, t_{86}=$ $2.68, p=.009 ; 90-10 / 3$ vs. $\left.80-20 / 2, t_{86}=5.08, p<.001\right)$ conferred performance improvements.

In the test/transfer phase, HVs compared with PSZ also selected the optimal stimulus more often $\left(F_{1,85}=10.26, p=\right.$ .002). Specifically, HVs outperformed PSZ on $90-10 / 3\left(t_{85}=\right.$ 2.68, $p=.009)$ and $80-20 / 1\left(t_{85}=2.51, p=.01\right)$, but not 80 $20 / 2\left(t_{85}=1.34, p=.19\right)$ and $90-10 / 1\left(t_{85}=1.64, p=.10\right)$ trials (Figure 2A). Nevertheless, PSZ and HVs performed above chance on all original pairs (all $p<.001$ ), suggesting that both groups had developed a preference for the optimal stimulus.

Subgroup analyses in LMD and MMD subgroups revealed no evidence for an effect of motivational deficit severity on acquisition pair accuracy during either experiment phase (Supplemental Results, Supplemental Figure S6).

\section{Performance on Novel Transfer Pairs}

The value difference tracking slope was greater for HVs than PSZ $\left(t_{82}=3.34, p=.001 ; 3 \mathrm{PSZ}\right.$ were removed due to limited choice variability) (see Supplemental Figure S7 for performance on every test/transfer phase pair for each diagnostic group) and remained significant when not correcting for a sidebias (i.e., logistic regression investigation correct choices as a function of value difference [optimal stimulus - suboptimal stimulus]; $\left.t_{82}=4.72, p<.001\right)$. In line with predictions, these data indicate that PSZ performance improved less as the difference in EV between two competing stimuli increased. Importantly, the group difference in the value difference tracking slope was driven by motivational deficit (avoltion/rolefunctioning and anhedonia/asociality subscales) severity (HVs vs. LMD, $t_{56}=1.78, p=.08 ; \mathrm{HVs}$ vs. MMD, $t_{62}=3.80, p<.001$, LMD vs. MMD, $t_{44}=0.65, p=.10$ ) (Figure $2 \mathrm{~B}$ ). These results 
suggest that the MMD subgroup specifically was poorer at integrating reward probability and magnitude. BPRS positive symptom scores were not associated with the value difference tracking slope (Spearman's $\rho=.15, p=.32$ ).

Focusing on selective trials matched for probability and magnitude, we observed a group $\times$ trial type interaction $\left(F_{1,85}=4.35, p=.04\right)$ (Figure 3 ). Post hoc analyses revealed that HVs performed better on magnitude discrimination than probability discrimination trials $\left(t_{37}=3.59, p=.001\right)$, while PSZ performed similarly on magnitude and probability discrimination trials $\left(t_{48}=0.73, p=.47\right)$ (Figure 3$)$. The difference between performance on magnitude- and probability-discrimination trials-that is, the difference between the advantage conferred by higher reward magnitude versus higher reward probability-highly correlated with the value difference tracking slope, suggesting that participants who performed better on magnitude discrimination trials overall performed better in the test/transfer phase (Spearman's $\rho=.56, p<.001$ ).

\section{Computational Modeling Analyses: Avolitional PSZ Overutilize Stimulus-Response Associations}

Mean hybrid-probability model parameter estimates for each diagnostic group are reported separately in Table 2, with only the mixing parameter differing between HVs and PSZ $\left(t_{85}=2.04, p=\right.$ .04) (see Supplemental Table S2 for individual model parameters). While a group difference (HV vs. PSZ) in the mixing parameter is in line with previous work (7), it did not survive

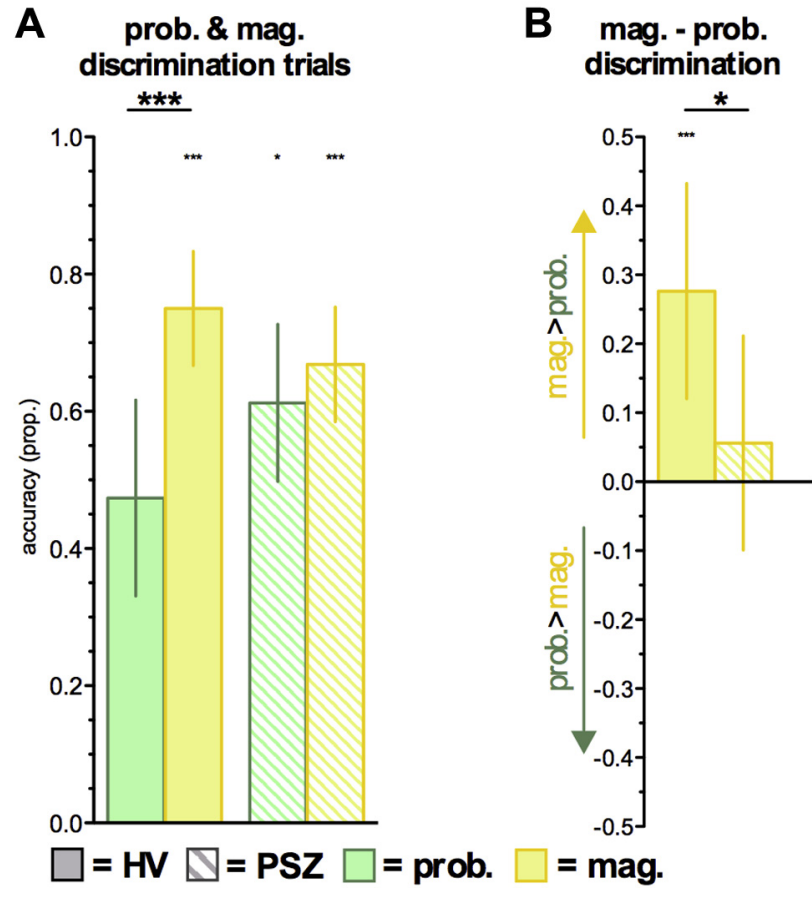

Figure 3. Performance on magnitude (mag) and probability (prob) discrimination trials during the test/transfer phase for each diagnostic group. (A) Performance on probability (90/1-80/1) and magnitude (90/3-90/1 and 80/2-80/1) discrimination trials. (B) The difference in performance on magnitude and probability trials. ${ }^{*} p<.05,{ }^{* \star *} p<.001$. Small asterisks above bars represent significance against chance. Error bars reflect $95 \%$ confidence intervals. HVs, healthy volunteers; PSZ, people with schizophrenia.
Table 2. Hybrid-Probability Model Parameters

\begin{tabular}{lcccc}
\hline & HVs $(n=38)$ & PSZ $(n=49)$ & $t$ & $p$ \\
\hline Critic Learning Rate $\left(\alpha_{\mathrm{c}}\right)$ & $0.49(0.42)$ & $0.32(0.37)$ & 1.95 & .35 \\
\hline Actor Learning Rate $\left(\alpha_{\mathrm{a}}\right)$ & $0.66(0.41)$ & $0.56(0.43)$ & 1.06 & .29 \\
\hline Q Learning Rate $\left(\alpha_{\mathrm{Q}}\right)$ & $0.29(0.31)$ & $0.23(0.32)$ & 0.86 & .39 \\
\hline Mixing Parameter $(m)$ & $0.64(0.34)$ & $0.48(0.39)$ & 2.04 & .04 \\
\hline Inverse Temperature $(\beta)^{a}$ & $0.34(0.41)$ & $0.29(0.42)$ & 0.50 & .62 \\
\hline
\end{tabular}

Values are $n(\mathrm{SD})$

$\mathrm{HVs}$, healthy volunteers; PSZ, people with schizophrenia

${ }^{a} \beta$ is inverse temperature multiplied by 100 .

correction for the number of parameters in the model. Crucially, however, the group difference in the mixing parameter was driven by motivational deficit (avolition/role-functioning and anhedonia/asociality subscales) severity (HVs vs. LMD, $t_{58}=$ $0.70, p=.48$, Cohen's $d=.19$; HVs vs. MMD, $t_{63}=2.69, p=.009$, Cohen's $d=.67$; LMD vs. MMD, $t_{47}=1.55, p=.13$, Cohen's $d=$ .44) (Figure 4) and correlated with Scale for the Assessment of Negative Symptoms total severity (Spearman's $\rho=-.29, p=.04$, $d f=48$ ) and Scale for the Assessment of Negative Symptoms Avolition scores specifically (Spearman's $\rho=-.28, p=.05$ ). The low mean mixing parameter (.40) suggests that MMD subgroups overutilized actor-critic-type learning, while LMD subgroups (.57) and HVs (.64) on average relied more on Q-learning. HV and MMD subgroups also marginally differed in $\alpha_{c}\left(t_{1,63}=1.99, p=\right.$ .052), suggesting that PSZ with motivational deficits updated their state value less in response to recent outcomes. BPRS positive symptom scores were not associated with the mixing parameter (Spearman's $\rho=-.03, p=.82$ ).

As predicted, the mixing parameter highly correlated with the value difference tracking slope in the entire sample, suggesting that greater reliance on Q-learning, in which choice values converge to their true expected values (16), was associated with better probability-magnitude integration (Spearman's $\rho=.39, p<.001)$. The mixing parameter additionally correlated with the difference between performance on magnitude- and probability-discrimination trials (Spearman's

\section{hybrid-probability model parameters}

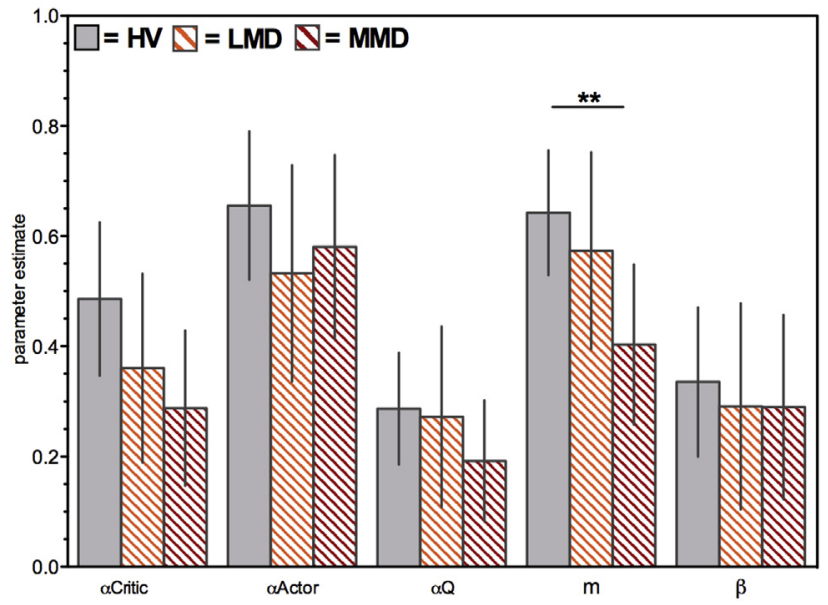

Figure 4. Hybrid-probability model parameters for healthy volunteers (HVs) and patients with less severe motivational deficit (LMD) and more severe motivational deficit (MMD). ${ }^{\star *} p<.01$. Error bars reflect $95 \%$ confidence intervals. 
$\rho=.38, p<.001)$. This finding is line with our demonstration of the actor-critic being insensitive to reward magnitude (see Model Selection and Comparison in the Supplement) and suggests that better discrimination of reward magnitude was associated with more EV-based learning.

These results suggest that increased reliance on stimulusresponse associations and/or decreased use of EV in PSZ with motivational deficits, as demonstrated by our hybridprobability model, is associated with impaired probabilitymagnitude integration and may be driven by reduced sensitivity to reward magnitude specifically.

\section{Hybrid-Probability Model Simulations}

Hybrid-probability model learning phase simulations closely approximated empirical data, with a predicted main effect of group on accuracy for $90-10 / 3\left(t_{85}=3.21, p=.002\right), 80-20 / 2$ $\left(t_{85}=3.52, p<.001\right), 80-20 / 1\left(t_{85}=2.28, p=.03\right)$ trials and a similar nonsignificant trend for $90-10 / 1\left(t_{85}=1.61, p=.11\right)$ trials (Figure 5A). Analyses of simulated data revealed either significant between-group differences or near-significant between-group differences on all acquisition pair test/transfer phase trials $\left(90-10 / 3, t_{85}=2.32, p=.02 ; 80-20 / 2, t_{85}=1.85\right.$, $\left.p=.07 ; 90-10 / 1, t_{85}=2.41, p=.02 ; 80-20 / 1, t_{85}=1.89, p=.06\right)$ (Figure $5 \mathrm{~A} ; n$ simulations $=20$ ).

Consistent with the empirical test/transfer phase data, simulations revealed numerically greater deficits in PSZ for easier choices (i.e., greater EV difference). Greater deficits for trials on which successful probability-magnitude integration decreases choice difficulty were also observed in PSZ relative to HVs (e.g., 90/3-80/1 and 90/3-20/1 ["1.9" and "2.5" in Figure 5B]). Crucially, the hybrid-probability model predicted a numerically smaller value difference tracking slope in MMD
A

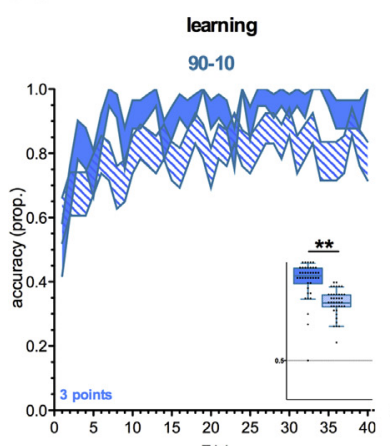

Acquisition Phase Pairs (simulated)

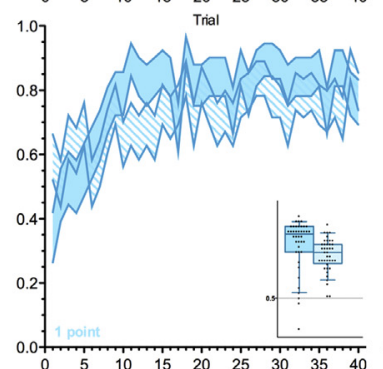

transfer
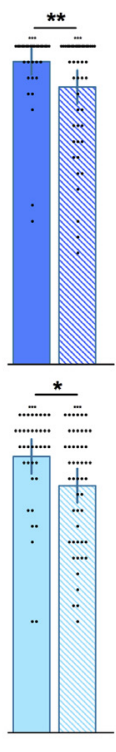

learning

80-20
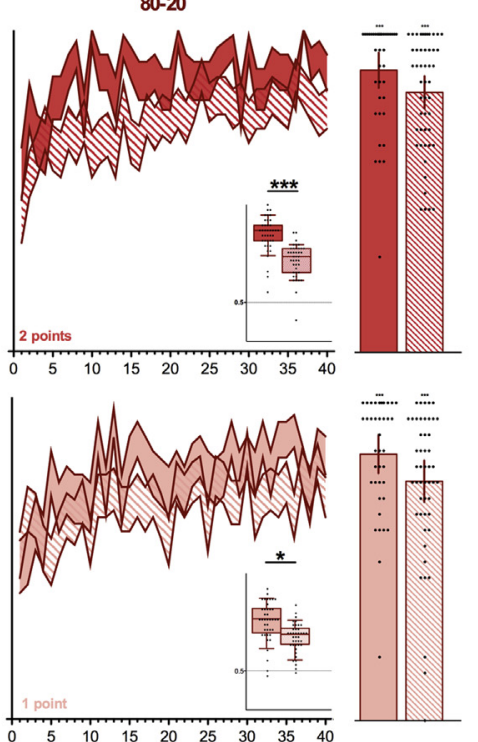

B Test/Transfer Phase (simulated)

novel pairs

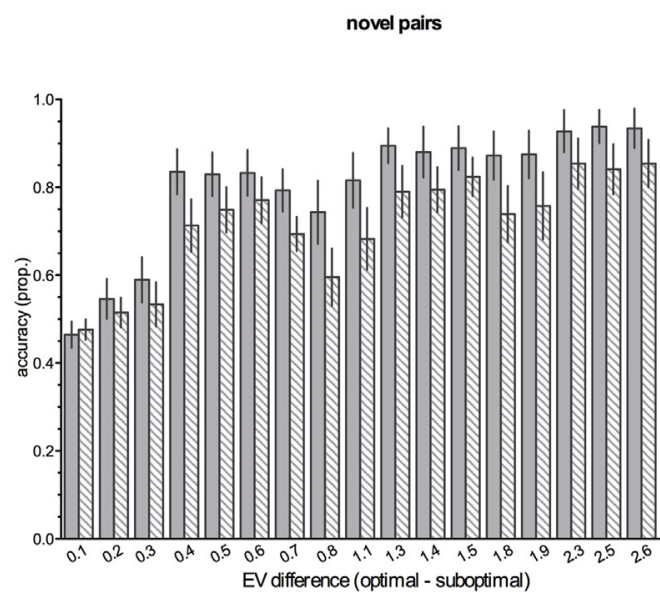

value difference
tracking slope $\frac{*}{* * *}$

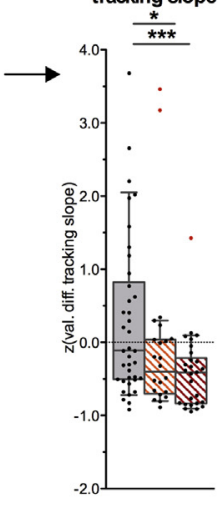

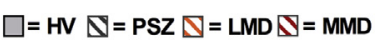

Figure 5. Hybrid-probability model simulations. (A) Simulated performance on acquisition phase pairs during the learning phase and test/transfer phase. (B) Simulated test/transfer phase performance on novel pairs presented for every value difference separately ("novel pairs") and the value difference tracking (two less severe motivational deficit $[\mathrm{LMD}]$ and one more severe motivational deficit [MMD] outliers marked in red). ${ }^{*} p<.05,{ }^{\star *} p<$ $.01,{ }^{\star \star *} p<.001$. Small asterisks above bars represent significance against chance. Error bars in panel (A) represent SEM and in (B) reflect $95 \%$ confidence intervals. EV, expected value; HVs, healthy volunteers; PSZ, people with schizophrenia. 
(HVs vs. LMD, $t_{56}=2.44, p=.02 ; \mathrm{HVs}$ vs. MMD, $t_{62}=3.44, p=$ .001 ; LMD vs. HMD, $t_{44}=1.29, p=.20$ ) (Figure 5B). Taken together, the hybrid-probability model could account for key aspects of the learning phase and test/transfer phase data, as well as highly specific performance deficits in PSZ with motivational deficits.

Associations with antipsychotic dosage, model fit, IQ scores, MATRICS performance, and subgroup analyses in poor performers and poorly fit subjects are reported in the Supplemental Results.

\section{DISCUSSION}

In contrast to many other RL paradigms, EV-based decision making in the current study relied on successful integration of reward probability and magnitude. PSZ were specifically impaired on trials with greater objective EV difference between two stimuli, as evidenced by the group difference in the test/ transfer phase value difference tracking slope, which was primarily driven by PSZ with motivational deficits. The inability to combine reward magnitude and probability in the service of generating adaptive estimates of EV is in line with a large body of previous work, including findings of performance deficits in PSZ on the lowa Gambling Task (10-12). Additionally, the finding of greater impairments for easier choices is consistent with a previously reported smaller value difference tracking slope in PSZ in the context of an $\mathrm{RL}$ paradigm where reward probability determined stimulus EV (7). Altogether, the current work reconfirms the notion that performance deficits in PSZ increase with demands placed on putative prefrontal processes involved in EV estimation, a rather robust finding in this population $(6,7,27,28)$.

Using a previously validated computational approach, we showed that outcome probability-magnitude integration deficits in PSZ with motivational deficits were driven primarily by increased reliance on valueless stimulus-associations (actorcritic), in lieu of EV-based decision making (Q-learning). Moreover, individual value difference tracking slopes correlated significantly with estimates of individual mixing parameters, which capture the balance between Q-learning and actor-critic-type learning, suggesting a systematic relationship between EV-based learning and probability-magnitude integration. Crucially, individual mixing parameters correlated significantly negatively with motivational deficit severity, thereby providing formal computational modeling evidence that impaired probability-magnitude integration in PSZ with motivational deficits can arise from overutilization of stimulusresponse associations.

The current results are noteworthy because they provide a first mechanistic explanation of complex EV estimation deficits in PSZ (with motivational deficits) that result from an inability to combine different dimensions of reward. Moreover, this work demonstrates that EV deficits in PSZ with motivational deficits are observable even when reward value subtly differs between stimuli, and not only when stimuli from diametrically opposed contexts (e.g., gain-seeking and/or loss-avoidance) are combined $(6,29)$. Selective associations with negative symptom severity moreover suggest that probability-magnitude integration deficits may play a role in the onset of motivational deficit severity. A failure to appropriately combine reward magnitude and probability into a single estimate of EV may lead to a decrease in perceived reward value. Underestimations of EV may change the trade-off between reward and effort cost, in line with findings that abnormal effort-cost computations are most pronounced in avolitional PSZ in conditions with high reward value (30-32). Thus, probability-magnitude integration deficits may reduce willingness to exert effort, thereby playing a role in the onset of avolition and anhedonia.

The framework of reduced EV-based learning and/or increased reliance on stimulus-response associations has been used previously to explain performance deficits in PSZ (with motivational deficits), such as insensitivity to gains $(6,29)$ and a reduced ability to update expectations in response to positive RPEs $(28,33,34)$. Gold et al. (6) also reported a selective reduction in the mixing parameter in PSZ with high motivational deficits during a gain-seeking and/or lossavoidance task. More recently, we previously employed this computational approach to explain increased contextdependent learning in PSZ (7). Although the mixing parameter did not correlate with motivational deficit severity in Hernaus et al. (7), the critic learning rate $\left(\alpha_{c}\right.$, which directly related to the magnitude of context-dependent learning) was selectively increased in individuals with more severe motivational deficits. The observation that various manifestations of disrupted processes in EV estimation coalesce in this single computational framework, and that parameters in this framework relate to motivational deficit severity, increases its generalizability and suggests potential for the computational hybrid model as a diagnostic tool for the detection of motivational deficits.

Interestingly, outcome probability-magnitude integration deficits seemed to be driven by underutilization of reward magnitude specifically. This interpretation is supported by the between-group difference in performance improvement owing to outcome magnitude versus probability increases (while holding the other constant). This contrast (Figure 3) correlated with the value difference tracking slope in the entire sample, suggesting that better performance on magnitude trials was associated with more efficient probability-magnitude integration. This observation goes hand in hand with evidence for increased Q-learning in HV, a framework in which magnitudedriven RPEs also update EV. Moreover, in model simulations, we demonstrated that reward magnitude was not used in the formation of response tendencies in the actor-critic architecture; this was the framework that could account for PSZ performance. Thus, performance and modeling evidence suggests that undervaluation of reward magnitude was specifically related to performance deficits in PSZ. This notion is line with reports on reward processing in anhedonia $(35,36)$ and with observations in PSZ with motivational deficits during a outcome probability-magnitude integration task (14).

Speculating on the neural mechanisms involved, preclinical work suggests that reward magnitude is encoded by, among other mechanisms, neurons in the basolateral amygdala and OFC (37-41). Moreover, lesions to the basolateral amygdala alter reward magnitude encoding (42) and value-based decision making (43-45) involving OFC. In contrast, tracking of reward probability may involve midbrain $(46,47)$ and ventral striatum $(41,48,49)$, although the latter has also been 
implicated in reward magnitude processing $(41,49)$. The specific inability to utilize reward magnitude information in combination with previously reported EV deficits in PSZ may thus point to a deficit in the basolateral amygdala-OFC projection or ventral striatum. This is consistent with ventral striatal lesions' being associated with performance deficits on a stimulus selection task (as employed in the current study), but not an action learning task, in nonhuman primates (50). This interpretation is further supported by reduced amygdalahippocampal RPE and value signals in PSZ (51), as well as abnormal OFC and striatum outcome and anticipation-related signals in the psychosis spectrum (52-55), the latter occasionally traveling with motivational deficit severity. Moreover, deficits in amygdala-OFC coupling have been implicated in the psychosis spectrum and are associated with symptom severity (56).

In light of abnormal striatal reward processing signals in PSZ (54), but relatively intact striatal RPE signals in medicated PSZ (57), however, performance deficits in our medicated PSZ sample could also be associated with a change in cortical topdown projections that influence $Q$ values represented by striatal neurons $(58,59)$. This view is supported by reduced OFC-striatal connectivity during effort-based decision making (60) and anterior cingulate cortex-striatal connectivity during probabilistic reversal learning (61) in PSZ with motivational deficits. A better understanding of neural mechanisms associated with probability-magnitude integration could be achieved by disambiguating the neural signals associated with reward magnitude, reward probability, and probabilitymagnitude integration in PSZ. Regardless of the exact neural mechanism involved, a reduction in RPE signals specifically and reward processing signals more generally in individuals with motivational deficits may be associated with a reduction in phasic dopamine release (62).

In conclusion, we provide formal evidence for the notion that failure to integrate reward probability and magnitude in PSZ with motivational deficits is associated with overreliance on the learning of valueless stimulus-response associations. Performance improvements associated with increases in reward magnitude, in combination with analyses using our computational hybrid model, suggest that such deficits may be specifically associated with an impairment in the ability to precisely and adaptively represent reward magnitude. The results presented here add to the generalizability of the computational hybrid model in capturing a broad range of EV estimation deficits in PSZ with motivational deficits.

\section{Limitations}

Only a limited number of trials were available to directly investigate probability and magnitude processing. Moreover, magnitude and probability were not fully orthogonalized in this experiment, slightly reducing the total number of trials that could be used to study reward probability. Importantly, however, the key aim of this study was to study probabilitymagnitude integration deficits in an $\mathrm{RL}$ context, rather than specific deficits in reward magnitude or probability processing. Moreover, a selective deficit in learning from reward magnitude is in line with previous work $(14,36)$ and was predicted and demonstrated by our computational modeling framework. As outlined previously (7), there is a possibility that the observed deficits in PSZ with motivational deficits may reflect a decrease in model-based processing. One advantage to using Qlearning to capture deficits in performance is that this algorithm does not require one to assume that participants rely on any model-based expectations. Future tasks should focus on teasing apart model-based predictions from EV (Q)-based learning in order to provide new insights into $R L$ deficits in PSZ. Finally, our sample consisted of chronic, medicated PSZ. While antipsychotic drug doses were not associated with any outcome measures, a follow-up investigation in antipsychoticnaïve PSZ could provide more information on symptomspecificity.

\section{ACKNOWLEDGMENTS AND DISCLOSURES}

This work was supported by the National Institute of Mental Health (Grant No. MH80066 [to JMG]).

We thank Benjamin M. Robinson for his contributions to the task design and data collection.

JAW, JMG, and MJF report that they perform consulting for Hoffman La Roche. JMG has also consulted for Takeda and Lundbeck and receives royalty payments from the Brief Assessment of Cognition in Schizophrenia. JAW also consults for NCT Holdings. The current experiments were not related to any consulting activity. All other authors report no biomedical financial interests or potential conflicts of interest.

\section{ARTICLE INFORMATION}

From the Department of Psychiatry and Neuropsychology (DH), School for Mental Health and Neuroscience, Maastricht University, Maastricht, the Netherlands; Maryland Psychiatric Research Center (DH, ECB, JKB, JMG, JAW), Department of Psychiatry, University of Maryland School of Medicine, Baltimore, Maryland; Department of Cognitive, Linguistic, and Psychological Sciences (MJF), and Department of Psychiatry and Brown Institute for Brain Science (MJF), Brown University, Providence, Rhode Island; and Institute for Psychology (ECB), University of Lübeck, Lübeck, Germany.

Address correspondence to Dennis Hernaus, Ph.D., Faculty of Health Medicine and Life Science, Maastricht University, P.O. BOX 616 (loc. Vijverdal), 6200MD Maastricht, the Netherlands; E-mail: dennis.hernaus@ maastrichtuniversity.nl.

Received Aug 9, 2018; revised Nov 8, 2018; accepted Nov 27, 2018.

Supplementary material cited in this article is available online at https:// doi.org/10.1016/j.bpsc.2018.11.011.

\section{REFERENCES}

1. Strauss GP, Waltz JA, Gold JM (2014): A review of reward processing and motivational impairment in schizophrenia. Schizophr Bull 40(Suppl 2):S107S116.

2. Fervaha G, Foussias G, Agid O, Remington G (2015): Motivational deficits in early schizophrenia: Prevalent, persistent, and key determinants of functional outcome. Schizophr Res 166:9-16.

3. Dickinson D, Bellack AS, Gold JM (2007): Social/communication skills, cognition, and vocational functioning in schizophrenia. Schizophr Bull 33:1213-1220.

4. Velligan DI, Kern RS, Gold JM (2006): Cognitive rehabilitation for schizophrenia and the putative role of motivation and expectancies. Schizophr Bull 32:474-485.

5. Fervaha G, Foussias G, Agid O, Remington G (2014): Motivational and neurocognitive deficits are central to the prediction of longitudinal functional outcome in schizophrenia. Acta Psychiatr Scand 130:290-299.

6. Gold JM, Waltz JA, Matveeva TM, Kasanova Z, Strauss GP Herbener ES, et al. (2012): Negative symptoms and the failure to represent the expected reward value of actions: Behavioral and computational modeling evidence. Arch Gen Psychiatry 69: 129-138. 
7. Hernaus D, Gold JM, Waltz JA, Frank MJ (2018): Impaired expected value computations coupled with overreliance on stimulus-response learning in schizophrenia. Biol Psychiatry Cogn Neurosci Neuroimaging 3:916-926.

8. Waltz JA, Gold JM (2016): Motivational deficits in schizophrenia and the representation of expected value. Curr Top Behav Neurosci 27:375-410.

9. Bechara A, Damasio AR, Damasio H, Anderson SW (1994): Insensitivity to future consequences following damage to human prefrontal cortex. Cognition 50:7-15.

10. Brown EC, Hack SM, Gold JM, Carpenter WT Jr, Fischer BA, Prentice KP, et al. (2015): Integrating frequency and magnitude information in decision-making in schizophrenia: An account of patient performance on the lowa Gambling Task. J Psychiatr Res 66-67: 16-23.

11. Brambilla P, Perlini C, Bellani M, Tomelleri L, Ferro A, Cerruti S, et al. (2013): Increased salience of gains versus decreased associative learning differentiate bipolar disorder from schizophrenia during incentive decision making. Psychol Med 43:571-580.

12. Kim MS, Kang BN, Lim JY (2016): Decision-making deficits in patients with chronic schizophrenia: lowa Gambling Task and Prospect Valence Learning model. Neuropsychiatr Dis Treat 12:1019-1027.

13. Brown JK, Waltz JA, Strauss GP, McMahon RP, Frank MJ, Gold JM (2013): Hypothetical decision making in schizophrenia: The role of expected value computation and "irrational" biases. Psychiatry Res 209:142-149.

14. Albrecht MA, Waltz JA, Frank MJ, Gold JM (2016): Probability and magnitude evaluation in schizophrenia. Schizophr Res Cogn 5:41-46.

15. Kasanova Z, Waltz JA, Strauss GP, Frank MJ, Gold JM (2011): Optimizing vs. matching: Response strategy in a probabilistic learning task is associated with negative symptoms of schizophrenia. Schizophr Res 127:215-222.

16. Watkins C, Dayan P (1992): Q-learning. Mach Learning 8:279-292.

17. Furuyashiki T, Gallagher M (2007): Neural encoding in the orbitofrontal cortex related to goal-directed behavior. Ann N Y Acad Sci 1121: 193-215.

18. Padoa-Schioppa C, Cai X (2011): The orbitofrontal cortex and the computation of subjective value: consolidated concepts and new perspectives. Ann N Y Acad Sci 1239:130-137.

19. Rich EL, Wallis JD (2016): Decoding subjective decisions from orbitofrontal cortex. Nat Neurosci 19:973-980.

20. Padoa-Schioppa C, Assad JA (2006): Neurons in the orbitofrontal cortex encode economic value. Nature 441:223-226.

21. Gottfried JA, O'Doherty J, Dolan RJ (2003): Encoding predictive reward value in human amygdala and orbitofrontal cortex. Science 301:1104-1107.

22. Rolls ET, McCabe C, Redoute J (2008): Expected value, reward outcome, and temporal difference error representations in a probabilistic decision task. Cereb Cortex 18:652-663.

23. Rescorla RA, Wagner AR (1972): A theory of Pavlovian conditioning: Variations in the effectiveness of reinforcement and nonreinforcement. In: Black AH, Prokasy WF, editors. Classical Conditioning II: Current Research and Theory. New York: Appleton-Century Crofts.

24. Joel D, Niv Y, Ruppin E (2002): Actor-critic models of the basal ganglia: New anatomical and computational perspectives. Neural Netw 15:535-547.

25. Collins AG, Frank MJ (2014): Opponent actor learning (OpAL): Modeling interactive effects of striatal dopamine on reinforcement learning and choice incentive. Psychol Rev 121:337-366.

26. Frank MJ, Claus ED (2006): Anatomy of a decision: Striatoorbitofrontal interactions in reinforcement learning, decision making, and reversal. Psychol Rev 113:300-326.

27. Waltz JA, Frank MJ, Robinson BM, Gold JM (2007): Selective reinforcement learning deficits in schizophrenia support predictions from computational models of striatal-cortical dysfunction. Biol Psychiatry 62:756-764.

28. Dowd EC, Frank MJ, Collins A, Gold JM, Barch DM (2016): Probabilistic reinforcement learning in patients with schizophrenia: Relationships to anhedonia and avolition. Biol Psychiatry Cogn Neurosci Neuroimaging 1:460-473.
29. Hartmann-Riemer MN, Aschenbrenner S, Bossert M, Westermann C, Seifritz E, Tobler PN, et al. (2017): Deficits in reinforcement learning but no link to apathy in patients with schizophrenia. Sci Rep 7:40352.

30. Culbreth AJ, Moran EK, Barch DM (2018): Effort-based decisionmaking in schizophrenia. Curr Opin Behav Sci 22:1-6.

31. Treadway MT, Peterman JS, Zald DH, Park S (2015): Impaired effort allocation in patients with schizophrenia. Schizophr Res 161: 382-385.

32. Gold JM, Strauss GP, Waltz JA, Robinson BM, Brown JK, Frank MJ (2013): Negative symptoms of schizophrenia are associated with abnormal effort-cost computations. Biol Psychiatry 74:130-136.

33. Waltz JA, Xu Z, Brown EC, Ruiz RR, Frank MJ, Gold J (2017): Motivational deficits in schizophrenia are associated with reduced differentiation between gain and loss-avoidance feedback in the striatum. Biol Psychiatry Cogn Neurosci Neuroimaging 3:239-247.

34. Deserno L, Heinz A, Schlagenhauf F (2017): Computational approaches to schizophrenia: A perspective on negative symptoms. Schizophr Res 186:46-54.

35. Huys QJ, Pizzagalli DA, Bogdan R, Dayan P (2013): Mapping anhedonia onto reinforcement learning: A behavioural meta-analysis. Biol Mood Anxiety Disord 3:12.

36. Vignapiano A, Mucci A, Ford J, Montefusco V, Plescia GM, Bucci P, et al. (2016): Reward anticipation and trait anhedonia: An electrophysiological investigation in subjects with schizophrenia. Clin Neurophysiol 127:2149-2160.

37. Smith BW, Mitchell DG, Hardin MG, Jazbec S, Fridberg D, Blair RJ, et al. (2009): Neural substrates of reward magnitude, probability, and risk during a wheel of fortune decision-making task. Neurolmage 44:600-609.

38. Bermudez MA, Schultz W (2010): Reward magnitude coding in primate amygdala neurons. J Neurophysiol 104:3424-3432.

39. Saez RA, Saez A, Paton JJ, Lau B, Salzman CD (2017): Distinct roles for the amygdala and orbitofrontal cortex in representing the relative amount of expected reward. Neuron 95:70-77.e73.

40. Burke SN, Thome A, Plange K, Engle JR, Trouard TP, Gothard KM, et al. (2014): Orbitofrontal cortex volume in area 11/13 predicts reward devaluation, but not reversal learning performance, in young and aged monkeys. J Neurosci 34:9905-9916.

41. Stoppel CM, Boehler CN, Strumpf H, Heinze HJ, Hopf JM, Schoenfeld MA (2011): Neural processing of reward magnitude under varying attentional demands. Brain Res 1383:218-229.

42. Rudebeck PH, Mitz AR, Chacko RV, Murray EA (2013): Effects of amygdala lesions on reward-value coding in orbital and medial prefrontal cortex. Neuron 80:1519-1531.

43. Lichtenberg NT, Pennington ZT, Holley SM, Greenfield VY, Cepeda C, Levine MS, et al. (2017): Basolateral amygdala to orbitofrontal cortex projections enable cue-triggered reward expectations. J Neurosci $37: 8374-8384$

44. Fiuzat EC, Rhodes SE, Murray EA (2017): The role of orbitofrontalamygdala interactions in updating action-outcome valuations in macaques. J Neurosci 37:2463-2470.

45. Rudebeck PH, Ripple JA, Mitz AR, Averbeck BB, Murray EA (2017): Amygdala contributions to stimulus-reward encoding in the macaque medial and orbital frontal cortex during learning. J Neurosci 37:21862202.

46. Bayer HM, Glimcher PW (2005): Midbrain dopamine neurons encode a quantitative reward prediction error signal. Neuron 47:129-141.

47. Bayer HM (2004): A Role for the Substantia Nigra in Learning and Motor Control. New York: New York University.

48. Abler B, Walter H, Erk S, Kammerer H, Spitzer M (2006): Prediction error as a linear function of reward probability is coded in human nucleus accumbens. Neuroimage 31:790-795.

49. Yacubian J, Sommer T, Schroeder K, Glascher J, Braus DF, Buchel C (2007): Subregions of the ventral striatum show preferential coding of reward magnitude and probability. Neuroimage 38:557-563.

50. Rothenhoefer KM, Costa VD, Bartolo R, Vicario-Feliciano R, Murray EA, Averbeck BB (2017): Effects of ventral striatum lesions on stimulus-based versus action-based reinforcement learning. J Neurosci 37:6902-6914. 
51. Gradin VB, Kumar P, Waiter G, Ahearn T, Stickle C, Milders M, et al (2011): Expected value and prediction error abnormalities in depression and schizophrenia. Brain 134:1751-1764.

52. Segarra N, Metastasio A, Ziauddeen H, Spencer J, Reinders NR, Dudas RB, et al. (2016): Abnormal frontostriatal activity during unexpected reward receipt in depression and schizophrenia: Relationship to anhedonia. Neuropsychopharmacology 41:2001-2010.

53. Waltz JA, Schweitzer JB, Gold JM, Kurup PK, Ross TJ, Salmeron BJ, et al. (2009): Patients with schizophrenia have a reduced neura response to both unpredictable and predictable primary reinforcers. Neuropsychopharmacology 34:1567-1577.

54. Radua J, Schmidt A, Borgwardt S, Heinz A, Schlagenhauf F, McGuire P, et al. (2015): Ventral striatal activation during reward processing in psychosis: A neurofunctional meta-analysis. JAMA Psychiatry 72:1243-1251.

55. de Leeuw M, Kahn RS, Vink M (2015): Fronto-striatal dysfunction during reward processing in unaffected siblings of schizophrenia patients. Schizophr Bull 41:94-103.

56. Anticevic A, Tang Y, Cho YT, Repovs G, Cole MW, Savic A, et al (2014): Amygdala connectivity differs among chronic, early course, and individuals at risk for developing schizophrenia. Schizophr Bull 40:1105-1116.

57. Culbreth AJ, Westbrook A, Xu Z, Barch DM, Waltz JA (2016): Intact ventral striatal prediction error signaling in medicated schizophrenia patients. Biol Psychiatry Cogn Neurosci Neuroimaging 1:474-483.

58. Samejima K, Ueda Y, Doya K, Kimura M (2005): Representation of action-specific reward values in the striatum. Science 310:1337-1340.

59. Clarke HF, Cardinal RN, Rygula R, Hong YT, Fryer TD, Sawiak SJ, et al. (2014): Orbitofrontal dopamine depletion upregulates caudate dopamine and alters behavior via changes in reinforcement sensitivity. J Neurosci 34:7663-7676.

60. Park IH, Lee BC, Kim JJ, Kim JI, Koo MS (2017): Effort-Based reinforcement processing and functional connectivity underlying amotivation in medicated patients with depression and schizophrenia J Neurosci 37:4370-4380.

61. Hernaus D, Xu Z, Brown EC, Ruiz RR, Frank MJ, Gold JM, et al. (2018): Motivational deficits in schizophrenia relate to abnormalities in cortical learning rate signals. Cogn Affect Behav Neurosci 18:1338-1351.

62. Maia TV, Frank MJ (2017): An integrative perspective on the role of dopamine in schizophrenia. Biol Psychiatry 81:52-66. 